Revue des patrimoines

$42 \mid 2020$

Imagerie numérique et patrimoine culturel :

représentation et transmission des connaissances

\title{
Numérisation et valorisation de maquettes anciennes de villes
}

Synthèse des projets

Digitisation and interpretation of old scale models of cities: Synthesis of the projects

Kévin Jacquot, Gilles Halin et Christine Chevrier

\section{OpenEdition}

Journals

Édition électronique

URL : http://journals.openedition.org/insitu/27738

DOI : $10.4000 /$ insitu. 27738

ISSN : 1630-7305

Éditeur

Ministère de la Culture

Référence électronique

Kévin Jacquot, Gilles Halin et Christine Chevrier, « Numérisation et valorisation de maquettes anciennes de villes », In Situ [En ligne], 42 | 2020, mis en ligne le 12 juin 2020, consulté le 12 janvier 2021. URL : http://journals.openedition.org/insitu/27738 ; DOI : https://doi.org/10.4000/insitu.27738

Ce document a été généré automatiquement le 12 janvier 2021.

\section{(c) $($ ) $\odot$}

In Situ Revues des patrimoines est mis à disposition selon les termes de la licence Creative Commons Attribution - Pas d'Utilisation Commerciale - Pas de Modification 4.0 International. 


\title{
Numérisation et valorisation de maquettes anciennes de villes
}

\author{
Synthèse des projets \\ Digitisation and interpretation of old scale models of cities: Synthesis of the \\ projects
}

Kévin Jacquot, Gilles Halin et Christine Chevrier

1 L'unité mixte de recherche "Modèles et simulations pour l'architecture et le patrimoine » (MAP, UMR 3495, CNRS / ministère de la Culture) mène depuis 2010 un programme de numérisation de maquettes anciennes de villes et notamment des plansreliefs. Ce programme s'attache à constituer, enrichir, harmoniser et analyser des données propres au médium qu'est la maquette de ville (histoire et techniques de fabrication des plans-reliefs, histoire des modes de représentation du territoire, etc.). Les données produites visent également à éclairer le contexte de la réalisation des maquettes de ville, la représentation du territoire français et de ses diversités à l'époque de leur fabrication (que cela soit du point de vue de l'architecture, de l'urbanisme, des paysages et des textes historiques, d'archives, etc.). Les plans-reliefs sont emblématiques d'une mémoire collective tant à l'échelle nationale que locale. La collection nationale des plans-reliefs présente de manière originale le récit de la formation du territoire de la France. Les plans-reliefs sont un exemple des efforts portés à la compréhension, à la représentation du territoire dans le but de mieux le défendre, l'aménager, l'administrer et de le valoriser. Les guerres de siège qui jalonnèrent le règne de Louis XIV révèlent aussi le rôle des villes fortifiées qui défendaient la France et leur importance future dans l'aménagement du territoire. Pour les villes concernées, c'est aujourd'hui l'occasion de se réapproprier un patrimoine local inscrit dans un cadre national. À l'échelle locale, seule la création de modèles virtuels mis en réseau avec d'autres maquettes numérisées de la collection permettrait aux villes de tirer parti de la force de la collection.

2 Le pouvoir d'évocation et de séduction des maquettes et de leurs homologues numériques en fait des outils pédagogiques de premier ordre pour expliquer les 
techniques de construction et les principes théoriques, pour enseigner l'histoire de l'édifice ou de l'architecture ou plus simplement, pour présenter une information touristique de qualité. La valorisation de la maquette par la création d'un modèle numérique sous la forme de représentations virtuelles fixes, animées ou interactives, par le biais des jeux vidéo notamment, peut agir comme un "activateur de curiosité ${ }^{1}$. Le modèle virtuel ne se substitue pas pour autant à la maquette, mais permet de la mettre en relation - en réseau - avec d'autres documents ou d'autres plans-reliefs virtuels. Pour des chercheurs, le plan-relief virtuel peut devenir le cœur d'un système d'informations patrimoniales pour l'accès et l'échange de données multimédias patrimoniales. Cela peut être également un environnement pour la simulation d'illumination, restitutions archéologiques, comportement structural, etc.

\section{Les maquettes anciennes de villes en Europe}

3 On distingue en général deux grands types de maquettes d'architecture, selon qu'elles précèdent ou non l'objet qu'elles représentent: elles sont alors rétrospectives ou opératives $^{2}$ a posteriori ou a priori ${ }^{3}$, etc. Les maquettes anciennes de villes de notre corpus sont toutes rétrospectives. Il est également possible d'affiner cette classification en considérant le synchronisme entre la ville représentée et sa représentation en maquette. Dans le cas des maquettes de stratégie militaire comme les plans-reliefs, ces deux éléments sont synchrones tandis que dans les maquettes de restitution archéologique comme les maquettes de Rome d'Italo Gismondi et de Paul Bigot ${ }^{4}$, de Liège de Gustave Ruhl-Hauzeur, de Genève d'Auguste Magnin, etc., la ville représentée n'existait plus au moment de leur réalisation. Les maquettes anciennes de villes sont des objets patrimoniaux riches, dotés d'une valeur historiographique majeure pour de nombreuses disciplines parmi lesquelles l'urbanisme, l'architecture, la cartographie ${ }^{5}$, la fortification, etc.

4 En Europe, l'époque moderne est un terreau fertile pour le développement de nombreuses collections de maquettes de villes fortifiées ${ }^{6}$. Au-delà de l'apport de la représentation en trois dimensions, elles ont un pouvoir d'évocation et de séduction ${ }^{7}$ que l'on retrouve dans les peintures panoramiques de Robert Barker (1839-1806) ou les dioramas de Louis Daguerre (1787-1851)8. L'échelle de la représentation rend «la totalité de l'objet moins redoutable [car] dans le modèle réduit la connaissance du tout précède celle des parties $»^{9}$. La perception du passage du temps est elle aussi influencée, il semble ralentir ${ }^{10}$. L'homme face à la maquette est alors omniscient, un démiurge ${ }^{11}$. Plus qu'un outil pédagogique, les maquettes sont des objets de prestige symbolisant la connaissance et la maîtrise du territoire pour les souverains européens.

5 La collection de plans-reliefs ${ }^{12}$ conservée à l'hôtel des Invalides à Paris et au Palais des Beaux-Arts de Lille est l'une des collections de maquettes de villes les plus importantes et les plus connues d'Europe ${ }^{13}$. Elle est constituée d'une centaine de maquettes à l'échelle 1:600 pouvant atteindre plusieurs dizaines de mètres carrés. Quelques ensembles de maquettes lui sont antérieurs, telles les collections bavaroises de Straubing (1568), Munich (1570), Landshut (1571), Ingolstadt (1572) et Burghausen (1574) réalisées par Jakob Sandtner ${ }^{14}$. Ces maquettes de places fortes implantées aux frontières entre le $\mathrm{xVI}^{\mathrm{e}}$ et le $\mathrm{xvIII}{ }^{\mathrm{e}}$ siècle sont aujourd'hui des témoins de la formation du territoire européen et sont par là même emblématiques d'une mémoire collective, tant à l'échelle européenne, nationale que locale. Une dizaine de plans-reliefs, ainsi que les 
maquettes de Rome, de Liège et les maquettes de Munich et de Straubing seront évoquées à nouveau plus loin puisqu'elles ont fait l'objet de projets de numérisation. De nombreuses autres maquettes de villes fortifiées furent produites au cours des siècles. Parmi celles étudiées ici, on peut citer les maquettes de Cadix (1779), Genève (1896), Liège (1910), ainsi que celles, pour la Suisse, de Soleure (1921) et Zurich (1930) réalisées par Hans Ferdinand Langmack (1881-1952).

\section{Des maquettes augmentées}

6 On reconnaît aux maquettes la capacité de faciliter la compréhension des objets qu'elles représentent par leur utilisation de la troisième dimension. La contrepartie, comme nous l'avons vu précédemment, est une mise à distance de l'observateur. Avant l'ère numérique, plusieurs techniques ont été utilisées pour atténuer cette distance.

\section{Instrumentation optique de maquettes}

7 En architecture, les techniques de représentation graphique basées sur les projections orthogonales et coniques imposent la maîtrise préalable d'un ensemble de règles graphiques permettant la lecture des documents. Avec les maquettes, cette connaissance n'est plus nécessaire, mais la bonne lecture de la représentation reste entravée par l'échelle. Différentes méthodes sont mises en œuvre pour atténuer la perception de l'échelle ou l'effet d'écrasement inhérent à la visualisation en plongée des maquettes de grande taille. Auguste Magnin, Paul Bigot et Italo Gismondi, respectivement auteurs des maquettes de Genève et de deux modèles de Rome, ont par exemple volontairement accentué les dimensions verticales de leurs maquettes pour compenser l'atténuation de la perception de la profondeur de l'observation en vue plongeante ${ }^{15}$.

Le développement d'instruments optiques, d'appareils photographiques et d'endoscopes en particulier ont permis d'atténuer les inconvénients liés à la perception de l'échelle. L'exploitation des techniques endoscopiques sur les maquettes a donné naissance à divers lecteurs de maquettes ${ }^{16}$, des appareils de vision directe, photographiques ou vidéographiques, tels que le maquettoscope de l'architecte Robert Auzelle $^{17}$, ou le relatoscope de Martin Vantreeck ${ }^{18}$ qui simulent le point de vue que pourrait avoir un observateur à l'échelle de la maquette. Malgré ces innovations, le sentiment de réalisme ne peut être total, car il reste entravé par des problèmes macrophotographiques liés à la faible profondeur de champ et à une lumière dure.

\section{Numérisation des maquettes anciennes de villes}

Le numérique a apporté de nouvelles solutions. À l'échelle internationale, une quarantaine de projets de numérisation de maquettes anciennes de villes a été menée. Une grande variété d'échelles et de superficies témoigne de la diversité des villes et des époques représentées [fig. 1]. Ces facteurs physiques jouent un rôle déterminant dans les différentes opérations ${ }^{19}$ menant à la numérisation de ressources culturelles. En raison de leurs caractéristiques physiques (échelle, dimensions, quantité, matériaux, etc.), les maquettes mettent en difficulté les techniques d'acquisition les plus récentes, peu efficaces à relever les objets de grande dimension avec un niveau de détail élevé. 


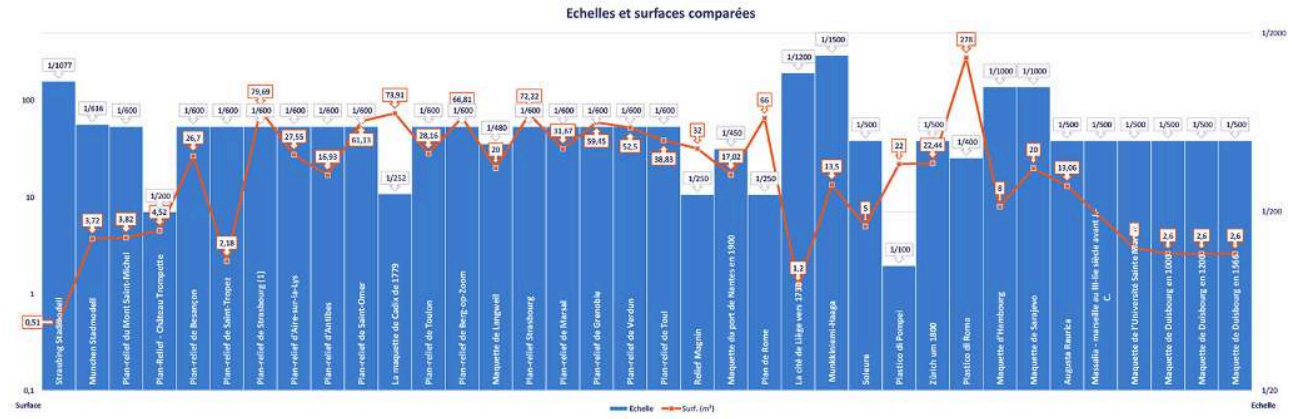

Diagramme comparant les surfaces et les échelles des maquettes des projets de numérisation étudiés.

Source : UMR MAP, 2018.

10 Qui plus est, les produits de l'acquisition 3D ne sont que des données dépourvues de toute sémantique. Pour que le relevé soit porteur de significations ${ }^{20}$, il doit subir des traitements de reconstruction géométrique visant à faire des données collectées un modèle de données du patrimoine bâti: un modèle numérique organisé sémantiquement et en trois dimensions des maquettes historiques [fig. 2].

Figure 2

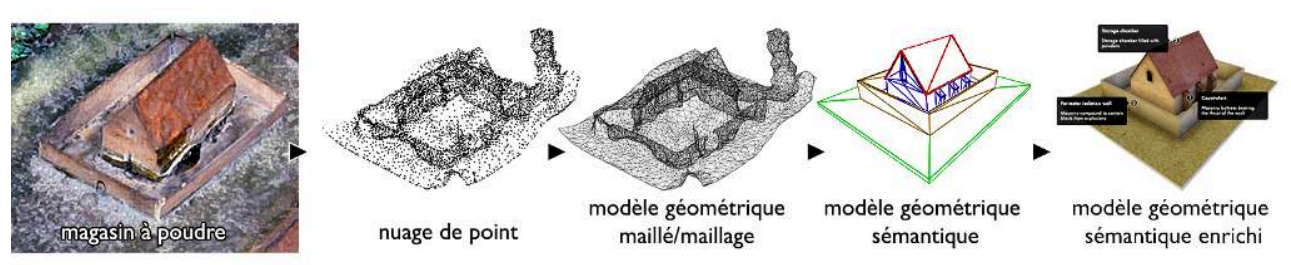

Principales étapes de la reconstruction et de la structuration d'un modèle numérique de magasin à poudre depuis son acquisition 3D jusqu'à son enrichissement sémantique.

Cliché : UMR MAP, 2018

\section{Collecte des données brutes à partir de maquettes anciennes}

11 Nous ne détaillerons pas les projets utilisant des données graphiques pour la création de la maquette virtuelle. Ces projets (Sarajevo, Straubing, Zurich) s'appuient sur des reportages photographiques dont les clichés sont utilisés pour une modélisation 3D réalisée manuellement par des infographistes. Il faut néanmoins s'attarder sur le projet portant sur le plan-relief de Verdun. C'est l'une des trois expériences conduites par le MAP sur les plans-reliefs et la seule qui utilise exclusivement les documents graphiques préparatoires et déjà numérisés de la maquette dans le processus de modélisation automatique du modèle numérique ${ }^{21}$. 
La première étape d'acquisition 3D est menée à l'aide de différentes techniques (lasergrammétrie, photogrammétrie manuelle ou par corrélation dense, lumière structurée). Les données produites à l'issue de la collecte se présentent sous la forme de nuage de points ou maillage [fig. 2]. Il faut ainsi attendre le début des années 2000 et la démocratisation des techniques d'acquisition 3D pour voir se développer les premiers essais de numérisation de maquettes anciennes de villes. La photogrammétrie est ainsi testée par le MAP-Crai ${ }^{22}$ et ICube ${ }^{23}$ sur le plan-relief de 1749 de Strasbourg. Mais aucun projet n'est lancé car les tests d'acquisition 3D sont jugés non satisfaisants ${ }^{24}$. La numérisation de maquettes répond en effet à des spécificités uniques. Les techniques et outils mis en œuvre doivent être suffisamment précis et maniables pour pouvoir relever des maquettes de grande dimension, mais qui représentent des éléments architecturaux. Les plans-reliefs (1:600) présentent ainsi des objets de quelques dizaines de centimètres pour les plus grands (cathédrale) à moins d'un millimètre pour les plus petits (menuiserie ou moulure par exemple). Qui plus est, les problèmes d'accessibilité aux zones centrales des maquettes et la haute densité d'objets dans les parties urbaines provoquent de nombreux effets de masque qui rendent compliqué l'acquisition de nombreux éléments.

Figure 3

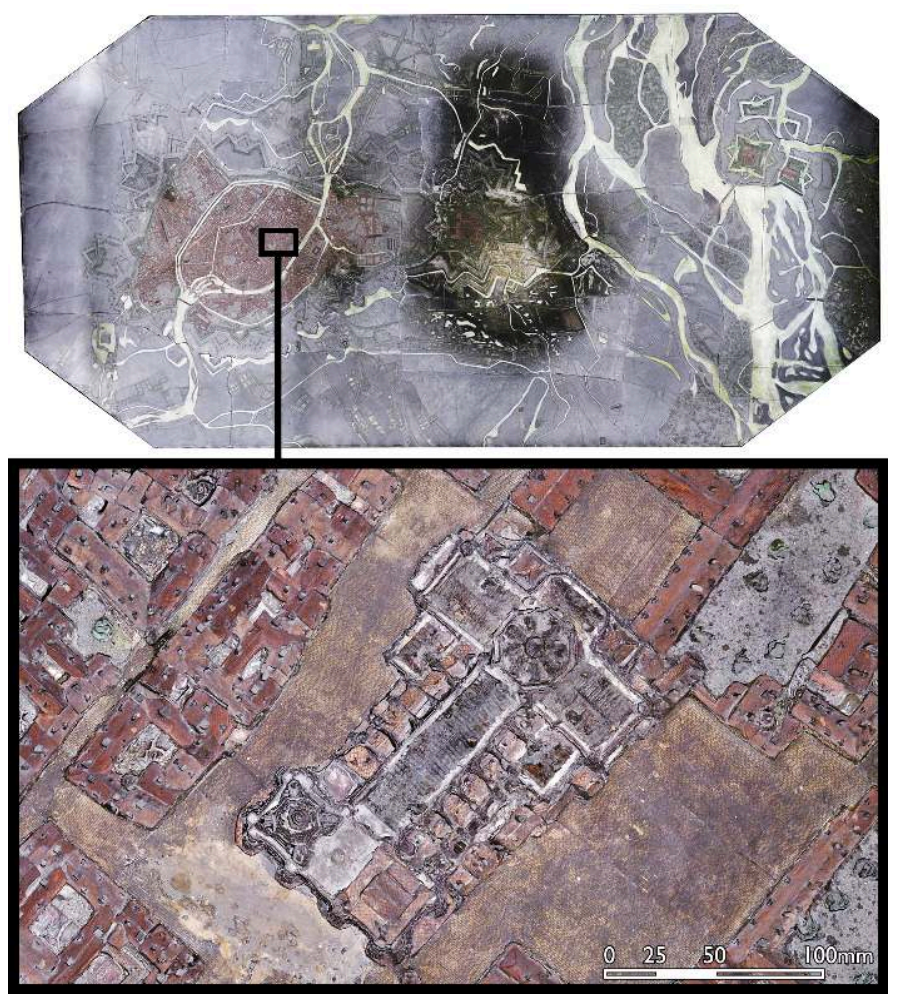

En haut : orthophotographie produite par photogrammétrie du plan-relief de 1728 de Strasbourg. En bas : vue de détail centrée sur la cathédrale Notre-Dame de Strasbourg.

Clichés: UMR MAP, INGEO, 2018.

13 Les projets utilisant la lasergrammétrie (Rome, Marsal, Nantes, Toul ${ }^{25}$ ) sont généralement antérieurs à 2010. Cette technique d'acquisition active a été utilisée dans les études patrimoniales dès le début des années 1990 et est restée pendant une vingtaine d'années une des techniques les plus courantes. Dans le cadre du projet Rome Reborn $^{26}$, plusieurs techniques d'acquisition actives ont ainsi été mobilisées: laser à 
temps de vol et à triangulation selon la précision requise et l'accessibilité, etc. La nécessité de déployer deux instruments de mesure traduit ici les limites de cette technique sur des objets comme les maquettes, qui ont pour singularité d'être des objets de plusieurs dizaines de mètres carrés affichant un niveau de détail très élevé, de l'ordre du millimètre. Peu d'outils peuvent satisfaire, seuls, cette double contrainte. Le coût de déploiement, l'encombrement de ces équipements et l'incapacité de ces techniques à collecter des données colorimétriques participent également grandement à la popularité de la photogrammétrie à corrélation dense.

Avec l'essor de la photogrammétrie à corrélation dense, de nouveaux projets sont menés dans lesquels techniques actives et passives sont associées pour se compléter (Duisbourg, Toul, Briançon). Outre la photogrammétrie, l'acquisition par lumière structurée permet à la même époque la collecte de données 3D et colorimétrique de plusieurs maquettes (Liège, Genève, Pékin, Hambourg). Les progrès en photogrammétrie, sur le plan matériel et logiciel, ont permis une démocratisation de cette technique. La photogrammétrie a été utilisée dans plus de la moitié des projets que nous avons étudiés. Plusieurs d'entre eux n'ont d'ailleurs pour objectif que la diffusion sur le web du modèle virtuel de la maquette sous la forme d'un maillage texturé que l'on peut survoler (Avignon, Cadix, Helsinki, Marseille, Nantes, Pompéi, Saint-Omer ${ }^{27}$ ), de vidéos (Marsal ${ }^{28}$ ) et la création d'une copie par impression 3D (Airesur-la-Lys $\left.{ }^{29}\right)$. Ces opérations sont aujourd'hui relativement accessibles grâce à l'automatisation de nombreux traitements appliqués aux données brutes (nettoyage, consolidation, colorisation, etc.). Néanmoins, les applications possibles de ces maillages sont limitées, car aucune interaction précise basée sur le contenu (bâtiment, rue, ouvrage, édifice, etc.) n'est possible.

Dans le cadre du projet «Urbania », l'acquisition par photogrammétrie a été réalisée par un partenaire privé possédant déjà une expérience des enjeux de l'acquisition 3D de maquettes de villes avec en particulier la numérisation du plan-relief d'Aire-sur-la-Lys en 2013. Le plan-relief de 1728 de Strasbourg mesure plus de $70 \mathrm{~m}^{2}$. Il est placé dans une vitrine équipée d'une passerelle mobile pour son entretien. L'opération de collecte des données 3D devant perturber le moins possible l'accessibilité des visiteurs, la méthode mise en œuvre s'appuie sur la présence de la passerelle [fig. 4]. Un système de repérage est mis en place pour assurer un recouvrement photographique de $70 \%$. La maquette a été subdivisée en zones de $20 \mathrm{~cm}^{2}$, chacune d'elles étant photographiée quatre fois de manière oblique afin de capter les façades et les emprises au sol de chaque bâtiment. Environ 5000 clichés ont ainsi été réalisés en cinq jours ${ }^{30}$. 


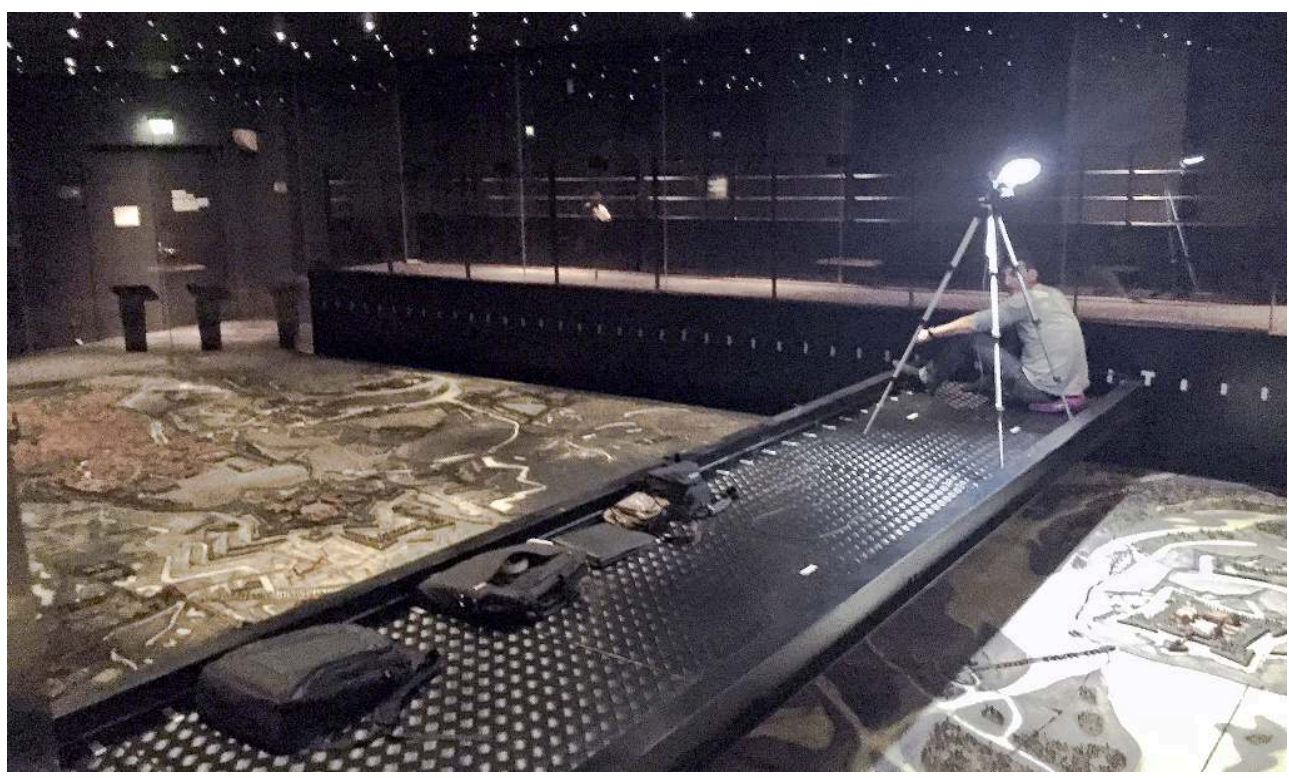

Dispositif pour l'acquisition photogrammétrique du plan-relief de 1728 de Strasbourg au Musée historique de Strasbourg.

Cliché : UMR MAP, Ingeo, 2018.

\section{Reconstruction numérique des maquettes anciennes}

Les traitements appliqués aux données collectées sur les maquettes anciennes de villes peuvent être distingués selon plusieurs critères parmi lesquels le degré d'automatisation dans les opérations de reconstruction géométrique et l'origine des données servant à enrichir visuellement la maquette virtuelle. La reconstruction géométrique a pour objectif de substituer au nuage de points ou au maillage un modèle mathématique, une instance d'un objet paramétrique [fig. 2], économe en données, précis, complet et enrichi sémantiquement. Dans la plupart des projets, cette segmentation géométrique utilise les données collectées comme support pour une reconstruction géométrique conduite par un opérateur humain à l'aide de logiciels d'infographie 3D couramment utilisés en DAO (dessin assisté par ordinateur) (Grenoble, Hambourg, Liège, Prague et les huit plans-reliefs numérisés par Google et la société Aloest pour l'exposition de 2012 «La France en relief $^{31}$ » ou n'est pas documentée (Briançon, Genève, Marsal, Munich, Nantes).

17 Les recherches pour automatiser la reconstruction géométrique des maquettes anciennes de villes s'inspirent de travaux menés à l'échelle de villes réelles et utilisant des données obtenues par acquisition passive ou active, aérienne et satellitaire. Ces approches sont dites «top-down» et utilisent a fortiori les données décrivant les toitures pour extrapoler la géométrie des bâtiments. Pour cela, elles recourent à des modèles paramétriques de bâtiments élaborés à partir de connaissances a priori sur l'objet à reconstruire ${ }^{32}$. Seuls les projets portant sur les maquettes de Duisbourg ${ }^{33}$, Soleure $^{34}$ et Hambourg ${ }^{35}$ mettent en œuvre des procédures de reconstruction automatiques. Les projets « Rome Reborn » et " Augusta Raurica ${ }^{36}$ » sont remarquables du fait de l'emploi d'un logiciel de génération procédurale de villes (CityEngine d'Esri) permettant la création automatique de plusieurs milliers de bâtiments répondant à des règles stylistiques précises. Une fois implémentées dans le logiciel, ces règles permettent de 
générer des bâtiments respectant le style architectural auquel ils appartiennent. Les maquettes numériques ainsi créées s'apparentent aux évocations telles que définies par Jean-Claude Golvin ${ }^{37}$ puisqu'elles cherchent à restituer une ambiance plutôt qu'une précision géométrique. De plus, la qualité géométrique et colorimétrique des modèles produits ne nécessite pas un niveau de détail élevé du fait de l'utilisation de Google Earth pour la diffusion des modèles.

Dans le cadre du projet "Urbania », le laboratoire ICube a développé une chaîne de traitements permettant d'extraire automatiquement les informations décrivant les plans de toitures et les emprises des cheminées des nuages de points. Ce traitement est actuellement achevé. Les informations sont aujourd'hui confiées au MAP-Crai qui a développé le logiciel eMaKet ${ }^{38}$, aboutissement des travaux de l'un des axes majeurs de recherche du laboratoire : la modélisation paramétrique pour le patrimoine bâti ${ }^{39}$. Le logiciel eMaKet permet le regroupement de primitives géométriques extraites par le laboratoire ICube en bâtiments. La procédure est automatique, mais des corrections manuelles demeurent possibles. À partir d'une bibliothèque de modèles de bâtiments paramétriques qui contient les typologies les plus courantes, un algorithme identifie parmi les primitives géométriques le type de bâtiment correspondant aux modèles implémentés avant d'ajuster une instance de ce même modèle aux dimensions relevées avec la texture obtenue par photogrammétrie. La conception d'eMaKet permet d'ajouter facilement de nouveaux modèles comme ceux spécifiques au plan-relief de Strasbourg (mansardes, fenêtres de toits, etc.). Enfin, les cours, rues, rivières, etc. sont automatiquement modélisées à l'aide de polygones prétexturés. Le MAP a également développé une approche pour reconstruire automatiquement les nombreuses fortifications bastionnées présentes sur les plans-reliefs. Ces ouvrages fortifiés sont conçus à partir de règles de construction qui peuvent être utilisées dans des approches de rétroconception ${ }^{40}$.

\section{Diffusion et interactivités des ressources numériques}

19 Appliquées aux maquettes anciennes de villes, les techniques de l'information et de la communication semblent se résumer essentiellement aux innovations de la fabrication assistée par ordinateur ${ }^{41}$ : impression 3D, découpe laser, etc. À l'autre bout du continuum numérique, la question de la diffusion fait l'objet de peu de recherches alors même que la présence du numérique et en particulier de maquettes augmentées dans les musées et centres d'interprétation tend à se banaliser. Hormis de rares projets de bornes muséales (Saint-Omer, Toul, Verdun) ou de maquette augmentée reposant sur l'enrichissement de maquettes anciennes par la projection de motifs lumineux permettant d'y associer une information visible sur un écran déporté (Nantes, Sarajevo, Straubing), la plupart des projets sont des applications de réalité virtuelle en ligne.

La diffusion peut ainsi répondre à l'enjeu d'accessibilité qui, réalisée sur internet et dans le navigateur, permet d'atteindre un public large. Des maquettes virtuelles de plusieurs projets nécessitent l'installation de programmes comme Acute3DViewer (Aire-sur-la-Lys, Avignon, Saint-Omer) ou Flash. Dans ce dernier cas, le devenir d'au moins deux projets (Genève, Zurich) est en sursis puisque Flash est appelé à disparaître en 2020. Avec l'arrivée à maturité du format de données HTML5 et de la spécification d'interface de programmation de 3D dynamique WebGL, de nouveaux outils de visualisation en ligne de contenus 3D apparaissent. Le visualiseur Sketchfab est très populaire (Cadix, Munich, Pompéi) et permet l'annotation des modèles virtuels (Cadix). 
Cependant, le degré d'interactivité de ces visualiseurs reste assez faible : la caméra virtuelle offre peu de liberté de mouvement et l'enrichissement sémantique reste pauvre par rapport à la richesse documentaire offerte par les maquettes anciennes de villes.

La conception de nouveaux outils satisfaisant les enjeux de diffusion de contenus 3D, enrichis visuellement, sémantiquement et interactifs est nécessaire, car aucune technique n'est à même de le faire actuellement. Deux approches se distinguent. La première repose sur des moteurs de jeu ou des kits de développement comme Unity et UDK, produits de l'industrie vidéoludique. Ces derniers figurent parmi les technologies mises en avant pour une diffusion optimale de contenus 3D par le programme européen 3D-ICONS. Bien que les modèles produits soient visuellement supérieurs aux systèmes d'information géographique, les moteurs de jeu ne permettent pas de stocker, de manipuler ou de diffuser des informations connexes aux modèles 3D sans recourir à des scripts pour accéder à des informations distantes. Les deux premiers projets du MAP se sont appuyés sur Unity [fig.5], tandis que le dernier projet a été l'occasion d'expérimenter un logiciel d'information géographique disposant de fonctionnalités 3D.

Figure 5

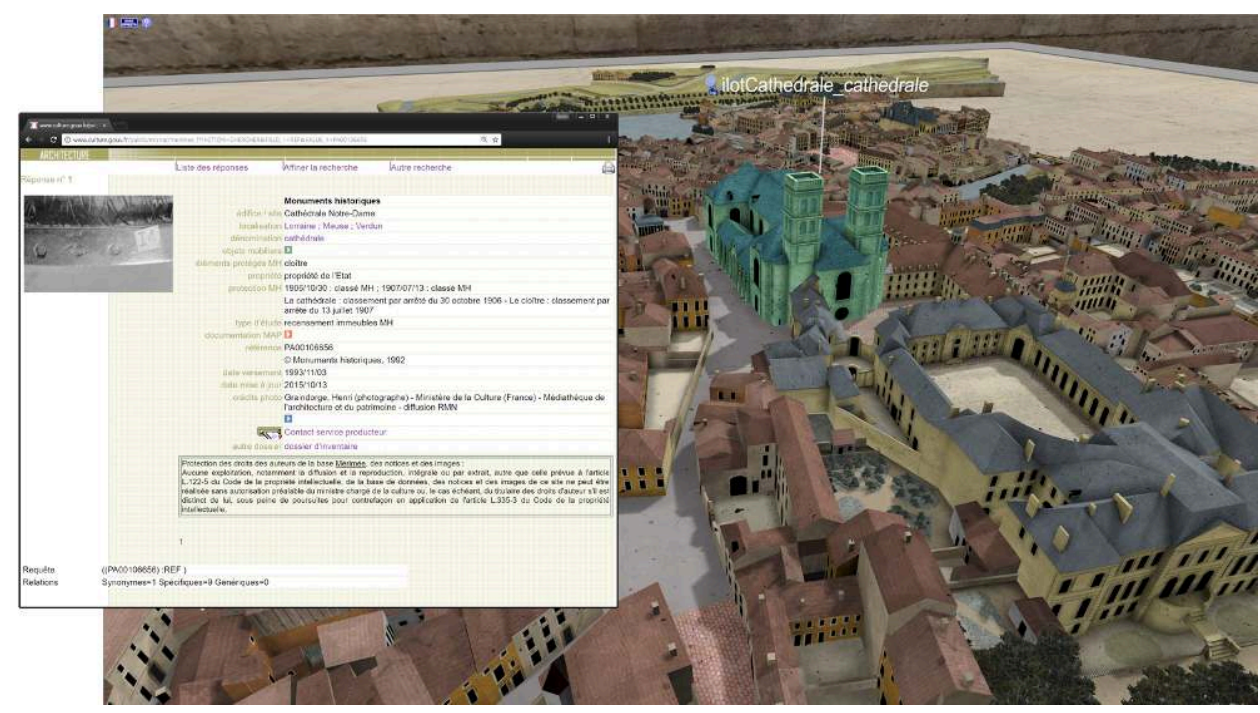

Capture d'écran de l'application finale pour le plan-relief de Verdun.

Cliché : UMR MAP, 2018.

La seconde approche repose donc sur l'utilisation de techniques issues de la géomatique et en particulier des systèmes d'information 3D web. La moitié de ces projets (Duisbourg, Hambourg, Rome, Soleure, les plans-reliefs numérisés par Aloest et Google) utilisent Google Earth, un système d'information qui présente des limitations rédhibitoires pour la gestion d'informations historiques. Beaucoup d'utilisateurs et de développeurs se sont tournés ces dernières années vers Google Earth. L'annonce en février 2015 du retrait de cet outil, de l'arrêt du développement de Google Map Engine et, in fine, de l'activité SIG professionnelle de Google, montre les limites de cette approche et la nécessité d'une approche SIG industrielle stable, engagée dans la durée auprès des utilisateurs et de la communauté scientifique. Dès 2004, la maquette du site archéologique d'Augusta Raurica - une cité romaine située à $10 \mathrm{~km}$ à l'est de Bâle, en 
Suisse - est au centre du projet «Dilas 3D GIS ». Développé au tout début des années 2000 , ce système d'information est parmi les premiers outils commercialisés permettant la création de SIG 3D web ${ }^{42}$ pour les maquettes anciennes de villes. D'autres projets (Genève, Liège, Prague) ont par la suite développé leur propre système d'information 3D à partir des maquettes, mais aucun de ces systèmes d'information n'apparaît accessible au grand public.

\section{Les maquettes numériques pour le patrimoine}

L'apport des maquettes anciennes de villes pour l'étude de l'histoire de l'architecture, des fortifications, de l'urbanisme, des modes de représentation, etc. démontre la richesse d'un patrimoine dont les différents projets de numérisation ont encore beaucoup de difficulté à rendre compte. Un premier projet pluridisciplinaire mené par le laboratoire MAP, le service régional de l'Inventaire de Lorraine, le musée des PlansReliefs sur le plan-relief de Toul et avec le concours financier du ministère de la Culture dans le cadre du programme national de numérisation, et de la Région Lorraine, a démontré l'intérêt de numériser des plans-reliefs, valorisés ensuite par les technologies de l'information et de la communication. La numérisation et la reconstruction virtuelle $\mathrm{du}$ plan-relief de Toul ont permis la mise en place d'un prototype de maquette 3D servant d'interface d'accès aux informations descriptives des objets représentés par le biais de liens vers la base Mérimée. Un second projet sur le plan-relief de Verdun a permis de mettre en œuvre une approche alternative en proposant une méthode basée non pas sur une acquisition 3D de la maquette, mais qui utilise les documents historiques et graphiques (plans et élévations) ayant servi à la construction de certains plans-reliefs.

Tandis que la numérisation de maquettes anciennes de villes bénéficie d'une grande diversité d'approches [fig. 6], les travaux menés dans le cadre du projet « Urbania » ont permis de conforter les méthodologies explorées par le MAP en matière de collecte, de traitement et diffusion des connaissances véhiculées par la maquette. Cette recherche, troisième projet du programme de numérisation de maquettes anciennes du MAP, est le cadre d'une collaboration entre des chercheurs en architecture, des historiens, des ingénieurs ainsi que deux sociétés privées spécialisées dans l'acquisition de données et dans les systèmes d'information 3D en ligne. 
Figure 6

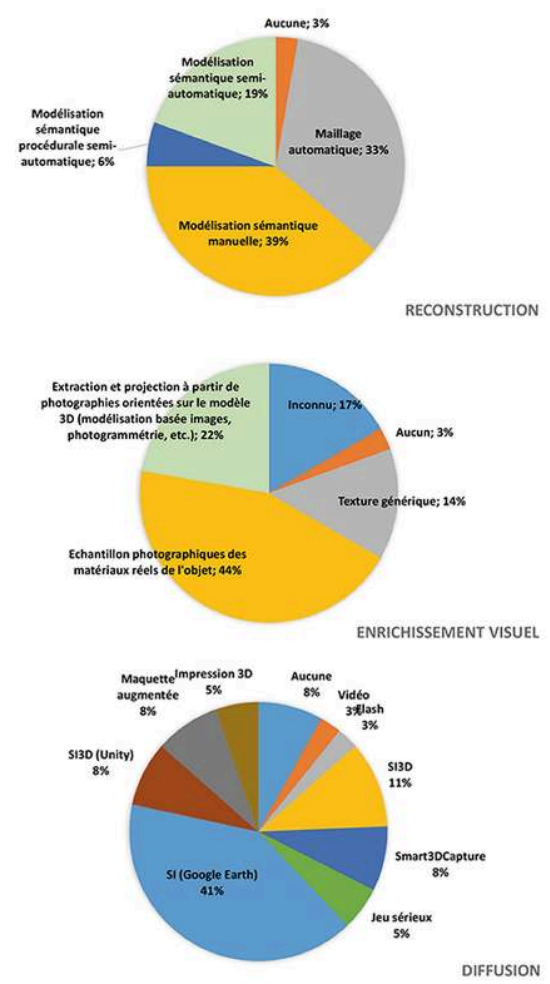

Diagrammes représentant les fréquences d'utilisation des différentes techniques mises en œuvre dans les projets de numérisation de maquettes.

Le pouvoir d'évocation et de séduction propre aux maquettes et l'attrait pour leurs homologues numériques en font des outils pédagogiques de premier ordre pour expliquer les techniques de construction et les principes théoriques, pour enseigner l'histoire de l'édifice ou de l'architecture, ou plus simplement pour présenter une information touristique de haut niveau. Le développement de systèmes d'information 3D utilisant les maquettes anciennes de villes comme interface est déterminant puisqu'il préfigure des rapprochements techniques et méthodologiques avec les maquettes numériques du domaine du BIM ${ }^{43}$, HBIM (Heritage BIM), SIG 3D, 4D, $\mathrm{nD}$, etc.

Ces dimensions viennent enrichir la notion de patrimoine telle qu'elle est abordée dans les projets de numérisation. Dans nos projets sur les plans-reliefs, la formalisation des connaissances architecturales, nécessaire à la reconstruction géométrique des maquettes, a permis de mettre en avant la structure duale existante entre l'objet construit et ses règles de construction, entre le tangible et l'intangible. Ces connaissances sur les dimensions immatérielles du patrimoine architectural sont encore peu valorisées et exploitées par les technologies de l'information et de la communication. Fortement interdisciplinaire, l'étude du processus de construction apporte pourtant un nouvel éclairage sur la construction, les types et techniques d'assemblage, etc., et permet d'interpréter les indices et vestiges du passé pour formuler des hypothèses de restitution de l'architecture. Elle offre également l'occasion de restituer le processus constructif de l'architecture, inscrit dans le temps pendant lequel s'érige le site ou le bâtiment. En mettant l'accent sur les processus et non la seule 
forme finie et statique, les études scientifiques, mais aussi les médiations résultantes sont plus justes et plus attractives.

\section{NOTES}

1. FLEURY Philippe \& MADELEINE Sophie, « Problématique d'une restitution globale de la Rome antique. Une visite interactive avec accès dynamique aux sources anciennes ", in VERGNIEUX Robert \& DELEVOIE Caroline (dir.), Virtual Retrospect 2007, actes du colloque éponyme (Pessac, 14-16 novembre 2007), Bordeaux, Ausonius, 2008, p. 56.

2. PÉROUSE DE MONTCLOS Jean-Marie, «Observation sur la viabilité et la fiabilité des maquettes ", Monumental, n² 21, « Maquettes d'architecture ", 1998.

3. ESTÉVEZ Daniel, « Dialogue entre maquette et modèle en architecture », in LÉVY Jean-Michel, Les Modèles, possibilités et limites, Paris, Éditions matériologiques, 2014, p. 77.

4. MADELEINE Sophie \& FLEURY Philippe, «Le "Plan de Rome" de Paul Bigot à l'université de Caen et son double virtuel: de l'objet patrimonial à l'outil scientifique ", In Situ. Revue des patrimoines, $\mathrm{n}^{\circ}$ 17, «Les patrimoines de l'enseignement supérieur», 2011 [en ligne], https:// journals.openedition.org/insitu/840 [lien valide en mai 2020].

5. BUISSERET David, « Modelling cities in early Modern Europe », in Envisioning the city. Six studies in urban cartography, Chicago / Londres, University of Chicago Press, 1998, p. 124-143.

6. VIGANÒ Marino, « Colecciones de modelos de plazas fuertes de los Borbones de Francia, España y Nápoles en el siglo XVIII », BSAA Arte, 2006, nº 72, p. 219-243.

7. GOLVIN Jean-Claude, Cours de Tunis. L'image de restitution et la restitution de l'image, Tunis, Icomos, 2005.

8. COMMENT Bernard, The Panorama, Londres, Reaktion Books, 2003 ; PESENTI CAMPAGNONI Donata \& TORTONESE Paolo (dir.), Les Arts de l'hallucination, Paris, Presses de la Sorbonne nouvelle, 2001.

9. LÉVI-STRAUSS Claude, La Pensée sauvage, Paris, Plon, 1962.

10. MITCHELL C. Thomas \& DAVIS Roy, "The perception of time in scale model environments ", Perception, 1987, vol. 16, nº 1, p. 5-16. DOI 10.1068/p160005.

11. ESTÉVEZ Daniel, «Petite mythologie de la maquette ", L'Architecture d'aujourd'hui, $\mathrm{n}^{\circ} 404$, « Maquettes d'architecture », 2014, p. 86-91.

12. WARMOES Isabelle, "Le musée des Plans-reliefs. Diversité des collections (1668-1940) et enjeux contemporains ", Artefact. Techniques, histoire et sciences humaines, $\mathrm{n}^{\circ} 7$, « Os, bois, ivoire et corne : l'exploitation des matières dures d'origine animale ", 2017, p. 223-230. Disponible en ligne, https://journals.openedition.org/artefact/1577 [lien valide en mai 2020].

13. BUISSERET David, « Modelling cities in early Modern Europe », art. cit., p. 125.

14. FROMMEL Sabine \& TASSIN Raphaël, Les Maquettes d'architecture: fonction et évolution d'un instrument de conception et de réalisation, Paris / Rome, Picard / Campisano, 2015.

15. GOLVIN Jean-Claude, Cours de Tunis. L'image de restitution et la restitution de l'image, Op. cit., p. 12. 16. LOYER François, "Pour bien lire une maquette d'architecture: le relatoscope», Communication et Langages, vol. 23, $\mathrm{n}^{\circ} 1,1974$, p. 56. DOI 10.3406/colan.1974.4130.

17. HAFFNER Jeanne \& GALISON Peter, «Modeling the social and the spatial. "Social Space" in postwar French social-scientific research », in HAFFNER Jeanne, The View from above. The science of social space, Cambridge / Londres, The MIT Press, 2013, p. 81-108. 
18. CENTRE D'ÉTUDES TECHNIQUES DE L'ÉQUIPEMENT, Principales techniques de visualisation. Prévoir pour mieux décider, Paris, ministère de l'Environnement et du Cadre de vie, 1980.

19. 3D-ICONS, 3D-ICONS Guidelines and case studies, Irlande, European Commission's ICT Policy Support Programme, 2014 [en ligne], https://pro.europeana.eu/files/Europeana_Professional/ Projects/Project_list/3D-ICONS/Deliverables/3D-

ICONS\%20Guidelines\%20and\%20Case\%20Studies.pdf [lien valide en mai 2020]; CONSORTIUM 3D SHS, Livre blanc du consortium 3D SHS, 2017.

20. MAZIÈRES François de \& GRUBERT Mireille (dir.), Le Relevé en architecture ou l'éternelle quête du vrai, actes des Journées internationales d'études (Cité de l'architecture \& du patrimoine, Paris, 5 et 6 novembre 2007), Lyon, Lieux dits, 2011.

21. CHEVRIER Christine, "Semiautomatic parametric modelling of the buildings on town scale models ». Journal on computing and cultural heritage, vol. 7, nº 4, p. 20:1-20:20. DOI 10.1145/2622609.

22. Centre de recherche en architecture et ingénierie, École nationale supérieure d'architecture de Nancy.

23. UMR 7357, Laboratoire de sciences de l'ingénieur, de l'informatique et de l'imagerie, Strasbourg.

24. CHEVRIER Christine, "Modélisations 3D et enrichissement sémantique pour un système d'information de plans-reliefs », Lille, 20 avril 2016.

25. BESNIER Jean-Marie, "Numérisation expérimentale d'un plan-relief », In Situ. Revue des patrimoines, $\mathrm{n}^{\circ} 16$, «Le patrimoine militaire et la question urbaine » [en ligne], https:// journals.openedition.org/insitu/397 [lien valide en mai 2020].

26. GUIDI Gabriele, FRISCHER Bernard, DE SIMONE Monica et al., «Virtualizing ancient Rome: 3D acquisition and modeling of a large plaster-of-Paris model of imperial Rome ", in BERALDIN Jean-Angelo, EL-HAKIM Sabry F. \& GRUEN Armin (dir.), Videometris VIII, Bellingham, SPIE, coll. « Proceedings of SPIE », 2005, p. 119-133.

27. http://www.patrimoines-saint-omer.fr/Le-territoire/Le-plan-relief-en-3D

28. https://youtu.be/mtMa-_VIwWE

29. https://youtu.be/9lv-J9tGWTo

30. MACHER Hélène, GRUSSENMEYER Pierre, LANDES Tania et al., « Photogrammetric recording and reconstruction of town scale models. The case of the Plans-Reliefs of Strasbourg ", The International Archives of the photogrammetry. Remote sensing and spatial information sciences, vol. XLII-2/W5, 2017, p. 489-495. DOI 10.5194/isprs-archives-XLII-2-W5-489-2017.

31. https://www.google.com/culturalinstitute/about/francerelief/

32. BRÉDIF Mathieu, «Modélisation 3D de bâtiments: reconstruction automatique de superstructures de toits et recalage cinétique de toits polyédriques prenant en compte la topologie », thèse de doctorat « Signal et image » soutenue à Télécom ParisTech (Paris), 2010.

33. PRZYBILLA Heinz-Jürgen, JEDRZEJAS Thomas, « Aufbau historischer 3D-Szenarien am Beispiel der mittelalterlichen Stadt Duisburg ", Photogrammetrie / Fernerkundung / Geoinformation, vol. 3, 2009, p. 199-207. DOI 10.1127/0935-1221/2009/0015.

34. NEBIKER Stephan, BARMETTLER Andreas, FISCHER Beat et al., « Vom physischen Stadtmodell zum interaktiven 3D-GIS. Anforderungen und Lösungsansätze am Beispiel der historischen Stadt Solothurn", Photogrammetrie/ Fernerkundung/ Geoinformation, vol. 3, 2009, p. 209-219. DOI 10.1127/0935-1221/2009/0016.

35. KELLER Friedrich, SÄNGER Jérome, KERSTEN Thomas et al., « Historical 4D city model of the Free and Hanseatic City of Hamburg. Automated generation and presentation within the Google Earth engine", Photogrammetrie / Fernerkundung/ Geoinformation, vol.3, 20113, p. 155-169. DOI 10.1127/1432-8364/2011/0080.

36. CHRISTEN M., BLASER Stefan, LOESCH Benjamin et al. «Prozedurale modellierung von historischen 3D-Stadtmodellen und deren Aufbereitung für 3D-visualisierungen in einer Smartphone-Applikation am Beispiel der römischen Stadt Augusta Raurica », Publikationen der 
deutschen Gesellschaft für Photogrammetrie, Fernerkundung und Geoinformation, vol. 25, 2016, p. 217 229.

37. GOLVIN Jean-Claude, Cours de Tunis. L'image de restitution et la restitution de l'image, Op. cit.

38. CHEVRIER Christine, "Semiautomatic parametric modelling of the buildings on town scale models », art. cit.

39. CHEVRIER Christine \& PERRIN Jean-Pierre, «Generation of architectural parametric components ", in Joining languages, cultures and visions. Proceedings of the 13th International CAAD futures conference, Montréal, Presses de l'université de Montréal, 2009, p. 105-118.

40. JACQUOT Kevin, CHEVRIER Christine, HALIN Gilles, "Reverse engineering of scale models using dataflow programming. Application to the fortification of Plans-Reliefs ", in 2013 Digital Heritage International Congress (DigitalHeritage), actes du colloque éponyme (Marseille, 28 oct.-1 nov 2013), vol. 1, Marseille, IEEE, 2013, p. 63-69. DOI 10.1109/DigitalHeritage.20 13.6743714 .

41. STAVRIĆ Milena, SIDANIN Predrag \& TEPAVCEVIĆ Bojan, Architectural scale models in the digital age. Design, representation and manufacturing, Vienne / New York, Springer, 2013.

42. WÜST Thomas, NEBIKER Stephan \& LANDOLT René, "Applying the 3D GIS DILAS to archaeology and cultural heritage projects requirements and first results ", International Archives of photogrammetry remote sensing and spatial information sciences, vol. 35, $\mathrm{n}^{\circ} 5,2004, \mathrm{p} .407-412$.

43. Building Information Modeling.

\section{RÉSUMÉS}

La maquette a été pendant longtemps le moyen de communication le plus efficace pour représenter l'architecture et la ville. Libérée des contraintes de la représentation graphique, la troisième dimension permet une lecture simplifiée de l'objet représenté. Au-delà de la question du remplacement de la maquette par des médiations numériques toujours plus réalistes et immersives, nous examinons ici les modes opératoires à l'œuvre dans les projets ainsi que les complémentarités entre maquettes physiques et numériques. Depuis une vingtaine d'années, un nombre croissant de projets mobilisent conjointement maquettes et sciences du numérique. L'archivage et la valorisation des maquettes sont des objectifs partagés par la plupart des projets, tandis qu'une minorité ambitionne la reconstitution d'environnements disparus et la création de systèmes d'information multidimensionnels permettant d'interfacer les données relatives aux maquettes.

Une quarantaine de projets faisant intervenir des maquettes anciennes de villes ont été étudiés parmi lesquels le programme de numérisation de plans-reliefs du MAP (UMR «Modèles et simulations pour l'architecture et le patrimoine »). Alors que l'acquisition 3D est l'étape première de la plupart des projets étudiés - et malgré les avancées en matière de collecte de données -, les propriétés exceptionnelles des maquettes constituent un premier obstacle. À l'autre bout de la chaîne de production, le produit final (système d'information $\mathrm{nD}$, copie physique, maquette augmentée, film, etc.) est également analysé pour dégager les usages et les formes de dialogue qui s'établissent entre maquette physique, modèle numérique et la connaissance relative à ces supports. Cet article synthétise les objectifs, les enjeux, les méthodes de production et de diffusion à l'œuvre dans ces approches et introduit le projet «Urbania », dernière recherche du MAP sur la numérisation de plans-reliefs. 
For a long time, scale models were the most effective means of communication to depict architecture and cities. Free from the constraints of the graphical representation, the third dimension allows a simplified reading of the depicted object. Beyond the question of replacing the model with increasingly realistic and immersive digital mediations, our communication investigates the processes at work in projects as well as the dialogue between physical and digital models. Over the past twenty years or so, a growing number of projects have brought together models and digital science. Archiving and valorisation of scale models are objectives shared by most projects, while a minority aspires to reconstitute past environments and create multidimensional information systems to interface the data relating to scale models.

Some forty projects involving old city models were studied, including MAP's digitalization program for plans-reliefs. While 3D acquisition is the first step in most of the projects studied and despite advances in data collection - the exceptional properties of scale models are a first challenge. At the other end of the production chain, the final product ( $\mathrm{nD}$ information system, physical replica, augmented scale models, film, etc.) is also analysed to identify the uses and forms of dialogues that are established between physical model, digital model and knowledge relating to these media. This paper synthesizes the objectives, challenges, production and dissemination methods at work in these approaches and introduces the 'Urbania' project, the latest research of MAP on the digitisation of relief planes.

\section{INDEX}

Mots-clés : Maquettes, système d'information géographique, numérisation 3D, plan-relief Keywords : scale models, geographic information system, 3D digitisation, plan-relief

\section{AUTEURS}

\section{KÉVIN JACQUOT}

Architecte, docteur en sciences de l'architecture, maître de conférences à l'École nationale supérieure d'architecture de Lyon, chercheur au laboratoire « Modèles et simulations pour l'architecture et le patrimoine culturel » (MAP-Aria, UMR 3495, CNRS / ministère de la Culture). kevin.jacquot@lyon.archi.fr

\section{GILLES HALIN}

Maître de conférence HDR de l'université de Lorraine, directeur adjoint du laboratoire « Modèles et simulations pour l'architecture et le patrimoine culturel » (MAP, UMR 3495, CNRS / ministère de la Culture), Nancy.

gilles.halin@crai.archi.fr

\section{CHRISTINE CHEVRIER}

Ingénieur de recherche, membre du Centre de recherche en architecture et ingénierie (MAP-Crai, UMR 3495, CNRS / ministère de la Culture), Nancy.

christine.chevrier@crai.archi.fr 